\title{
Diastolic function
}

Michael J. Lanspa, Troy D. Olsen, Emily L. Wilson, Mary Louise Leguyader, Eliotte L.

Hirshberg, Jeffrey L. Anderson, Samuel M. Brown, Colin K. Grissom

\section{Source}

MichaelJ. Lanspa, Andrea R. Gutsche, Emily L. Wilson, Troy D. Olsen, Eliotte L. Hirshberg, Daniel B. Knox. (2016). Application of a simplified definition of diastolic function in severe sepsis and septic shock. Crit Care, vol. 20 (1)

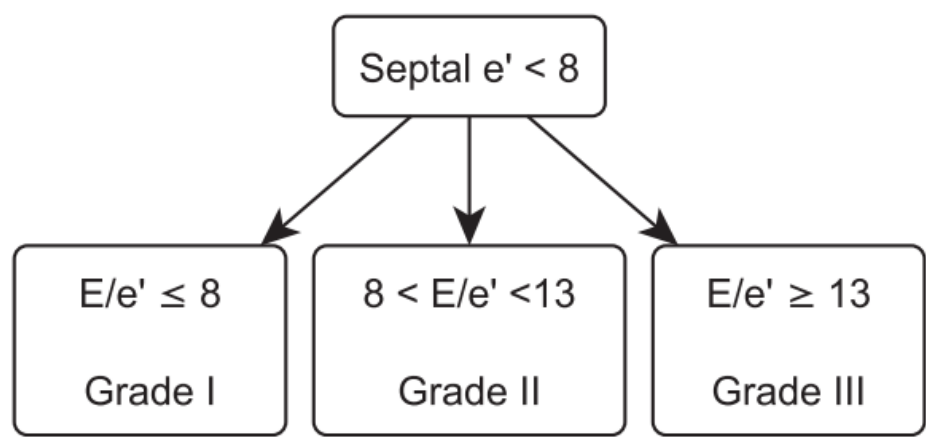

Simplified definition of diastolic dysfunction 